\title{
Un modèle micromécanique pour la nucléation de fissures en fatigue polycyclique
}

\author{
Vincent Monchiet, Éric Charkaluka et Djimedo Kondo \\ Laboratoire de Mécanique de Lille (LML), UMR 8107, 59655 Villeneuve d'Ascq, France
}

Reçu 15 mars 2007, accepté le 7 juin 2007

\begin{abstract}
Résumé - Une approche multiéchelle de la fatigue multiaxiale en endurance illimitée est proposée. Elle vise à rendre compte des mécanismes couplés de plasticité et d'endommagement qui surviennent le long des Bandes de Glissement Persistantes (BGP). L'endommagement, qui est couplé avec la plasticité, est modélisé comme la conséquence de la croissance de microcavités le long des BGP. Le critère de fatigue macroscopique proposé correspond à une condition de nucléation d'une fissure à l'interface BGP/matrice, et est obtenu pour le schéma d'homogénéisation de Kröner. On montre le rôle de la contrainte moyenne et de la pression hydrostatique en fatigue à grand nombre de cycles. Cette dépendance est ici liée à la prise en compte des micromécanismes d'endommagement. Enfin, on présente quelques illustrations dans le cas particulier des trajets de chargement affines. Des comparaisons du critère obtenu avec des résultats expérimentaux montrent la pertinence de l'approche.
\end{abstract}

Mots clés : Fatigue / micromécanique / endurance illimitée / BGP / endommagement

Abstract - A micromechanical model for crack nucleation in high cycle fatigue. A multiscale approach of multiaxial fatigue in unlimited endurance is proposed. It allows to take into account coupled plasticity and damage mechanisms which occur at the scale of Persistent Slip Bands (PSB). The damage is modelled as the consequence of microvoids growth along PSBs. The proposed macroscopic fatigue criterion, which corresponds to microcracks nucleation at the PSB-matrix interface, is derived by using Kröner homogenization scheme. The role of a mean stress and of the hydrostatic pressure in high cycle fatigue is shown. This dependence is related to the damage micro-mechanisms. Finally, some illustration concerning the particular case of affine loadings is presented. Comparisons of the obtained criterion with experimental data shown the relevance of the approach.

Key words: Fatigue / micromechanics / unlimited endurance / PSB / damage

\section{Introduction}

Il est largement admis aujourd'hui que la rupture par fatigue des pièces métalliques est le résultat de phénomènes microscopiques complexes qui apparaissent à l'échelle des grains sous sollicitations cycliques. En particulier, un rôle majeur est attribué à la microplasticité (due aux mouvements des dislocations) ainsi qu'aux processus d'endommagement par croissance de microfissures $[1,2]$. L'objectif de cette étude est la définition d'un critère de fatigue à grand nombre de cycles (ou fatigue polycyclique), fondé sur les mécanismes physiques microscopiques entraînant endommagement et fissuration; on s'intéressera essentiellement à la question de la détermination d'un seuil d'amorçage d'une fissure de fatigue.

\footnotetext{
a Auteur pour correspondance :

eric.charkaluk@univ-lille1.fr
}

On rappelle tout d'abord qu'il existe de nombreux critères de fatigue polycyclique à caractère phénoménologique (cf. notamment [3,4] ou [5]). Les premiers travaux véritablement basés sur des observations microscopiques disponibles sont ceux d'Orowan [6] puis de Dang Van [7] dont le grand intérêt est d'être fondés sur une analyse des phénomènes de microplasticité mis en évidence ou confirmés par d'autres auteurs. Cette approche multiéchelle a été reprise et formalisée par la suite par Papadopoulos [8]. Le cadre théorique ainsi établi repose sur une modélisation de la microplasticité et l'utilisation des théorèmes d'adaptation élastique afin d'établir un critère de non amorçage de fissures. On notera cependant que, dans ces approches à vocation multiéchelle, la prise en compte des phénomènes de microendommagement n'est pas explicite.

On propose dans cette étude de formuler une approche de la fatigue polycyclique basée sur un couplage entre les mécanismes de plasticité et d'endommagement à l'échelle 
des grains les plus sollicités. Ces micromécanismes d'endommagement, qui ont lieu le long des bandes de glissement intense, sont supposés de deux types : il s'agit, d'une part, de la nucléation de microcavités par accumulation de défauts ponctuels de type lacunaire créés par annihilation des dislocations, et, d'autre part, de la croissance de ces microcavités sous l'action combinée de la déformation plastique et des efforts de pression. Dans le cas d'une matrice de von Mises cette croissance est généralement décrite à l'aide de modèles d'endommagement ductile, classiquement définis sous chargements monotones $[9,10]$. Afin de décrire la croissance de ces microcavités le long des BGP, on propose d'adapter ici l'analyse limite de Gurson [10] développée pour la sphère creuse parfaitement plastique et régie par le critère de von Mises. La sphère creuse considérée alors ici représente en fait une cellule élémentaire du milieu monocristallin contenant des microcavités. La prise en compte de l'écrouissage, essentielle dans la perspective d'une application aux chargements cycliques, et donc pour la fatigue, sera décrite. À ce propos, la démarche s'appuie sur les travaux de Leblond et al. [11], adaptés et développés ici pour la plasticité cristalline. Le critère d'amorçage adopté correspond à une valeur critique du dommage à l'échelle des BGP.

En vue de la transposition du critère de fatigue à l'échelle macroscopique, un passage micro-macro est effectué en utilisant le schéma autocohérent de Kröner [12]. Enfin, une expression analytique du critère de fatigue sera explicitée dans le cas des trajets de chargement macroscopiques affines et déphasés. Afin d'évaluer la pertinence de l'approche proposée, des comparaisons du critère avec des résultats expérimentaux issus de la littérature seront proposées en dernière partie de l'article.

\section{Principes de base de la modélisation proposée}

\subsection{Comportement plastique cyclique à l'échelle des grains}

On propose dans cette section de décrire les micromécanismes de plasticité à l'échelle des grains. Ceux-ci se caractérisent par l'activation de systèmes de glissement. Dans le cadre de la fatigue polycyclique, pour les structures de type CFC, l'hypothèse de glissement simple est couramment adoptée $[7,8]$. Cette hypothèse se justifie par le fait que les amplitudes de contraintes restent faibles en fatigue à grand nombre de cycles, puisque en dessous du seuil de plasticité macroscopique.

On note par $\sigma$ et $\varepsilon$ respectivement les tenseurs de contrainte et de déformation à l'échelle locale. On adopte une décomposition additive de la déformation totale, $\varepsilon$, en une déformation élastique, $\varepsilon^{\mathrm{e}}$, et une déformation plastique, $\varepsilon^{\mathrm{p}}$ telle que : $\varepsilon=\varepsilon^{\mathrm{e}}+\varepsilon^{\mathrm{p}}$. Le glissement plastique, sur le système de glissement activé, est défini par $\gamma=2 \varepsilon^{\mathrm{p}}: \boldsymbol{\Delta}$, où $\boldsymbol{\Delta}$ est le tenseur d'orientation du système de glissement considéré, défini par :

$$
\Delta=\underline{n} \stackrel{s}{\otimes} \underline{m}=\frac{1}{2}(\underline{n} \otimes \underline{m}+\underline{m} \otimes \underline{n})
$$

dans lequel $\underline{n}$ désigne la normale au plan de glissement activé et $\underline{m}$ la direction du glissement. Le comportement plastique $\overline{\mathrm{d}} \mathrm{u}$ monocristal met en jeu la contrainte de cisaillement $\tau=\boldsymbol{\sigma}: \boldsymbol{\Delta}$ et le glissement plastique $\gamma$. De manière classique, ce comportement est régi par la loi de Schmid :

$$
|\tau-X|-\tau_{0}-R=0
$$

$X$ et $R$ sont respectivement les variables d'écrouissage cinématique et isotrope. Une loi linéaire pour l'écrouissage cinématique et pour l'écrouissage isotrope a été adoptée :

$$
X=c \gamma ; \quad R=R_{0} \gamma_{\text {cum }}
$$

$c$ et $R_{0}$ sont les modules d'écrouissage et $\gamma_{\text {cum }}=\int_{0}^{t}|\dot{\gamma}| \mathrm{d} t^{\prime}$ le glissement plastique cumulé.

\subsection{Prise en compte de l'endommagement}

\subsubsection{Cas de la plasticité parfaite}

Conformément à ce qui a été déjà précisé en introduction, la modélisation multiéchelle repose sur les mécanismes irréversibles qui altèrent de manière définitive la microstructure. Dans le cadre des structures de type CFC et des chargements cycliques, la localisation de la déformation plastique s'accompagne également d'un mécanisme d'annihilation de dislocations qui est à l'origine de l'accumulation de défauts ponctuels de type lacunaire le long des BGP. Un modèle phénoménologique pour la production de défauts par annihilation a été proposé par Essmann et al. [5]; la porosité induite par ce mécanisme, que l'on note $f_{\mathrm{a}}$, est donnée par :

$$
f_{\mathrm{a}}\left(\gamma_{\text {cum }}\right)=A_{0}\left[k_{\mathrm{a}} \gamma_{\text {cum }}-1+\exp \left(-k_{\mathrm{a}} \gamma_{\text {cum }}\right)\right]
$$

où $A_{0}$ et $k_{\mathrm{a}}$ sont deux paramètres du modèle. Le mécanisme d'annihilation, ainsi décrit, est uniquement piloté par le glissement plastique. Il ne peut donc rendre compte du rôle important de la pression hydrostatique sur le critère de fatigue. L'idée consiste alors à rechercher un second mécanisme d'endommagement, conjointement à celui que nous venons de décrire, et qui fait intervenir explicitement la pression hydrostatique.

Ces mécanismes d'annihilation entraînant la nucléation de cavités de type lacunaire, celles-ci sont susceptibles de croître sous l'action combinée de la déformation plastique de la matrice environnante et des efforts de pression, tel qu'il a été mis en évidence par Rice et Tracey [9] sous chargements monotones. On se propose donc d'introduire la variable $f_{\mathrm{g}}$ correspondant à la porosité induite par ce second mécanisme. On définit alors la porosité totale $f$, comme la somme des porosités $f=f_{\mathrm{a}}+f_{\mathrm{g}}$. La croissance des cavités induisant des variations de volume, on introduit une déformation plastique résultant de l'expansion de la cavité; ceci implique que la déformation plastique à l'échelle du grain s'écrit :

$$
\varepsilon^{\mathrm{p}}=\gamma \boldsymbol{\Delta}+\varepsilon_{\mathrm{h}}^{\mathrm{p}} \mathbf{1}
$$

où la partie hydrostatique de la déformation plastique $\varepsilon_{h}^{p}$ due à la croissance des cavités peut être reliée à $f_{\mathrm{g}}$ en 
utilisant la conservation de la masse (en considérant de faibles porosités) :

$$
\dot{f}_{\mathrm{g}}=(1-f) 3 \dot{\varepsilon}_{\mathrm{h}}^{\mathrm{p}} \simeq 3 \dot{\varepsilon}_{\mathrm{h}}^{\mathrm{p}}
$$

pour laquelle la déformation élastique de la matrice solide a été supposée négligeable.

On suppose alors qu'une condition suffisante de non rupture est obtenue lorsqu'aucune fissure de la taille d'un grain ne peut être observée. Une approche locale de la fatigue à grand nombre de cycles consiste alors à considérer une valeur critique de la porosité, notée $f_{\mathrm{c}}$ et en dessous de laquelle il n'y a pas d'amorçage de fissures. Le critère de fatigue qui s'en déduit s'écrit donc :

$$
f=f_{\mathrm{a}}\left(\gamma_{\text {cum }}\right)+f_{\mathrm{g}}\left(\varepsilon_{\mathrm{h}}^{\mathrm{p}}\right)=f_{\mathrm{c}}
$$

On remarquera qu'il est fonction de $\gamma_{\text {cum }}$ et de $\varepsilon_{\mathrm{h}}^{\mathrm{p}}$ et généralise donc, en quelque sorte, les approches considérant une valeur critique de la déformation plastique cumulée $\gamma_{\text {cum }}$ (critère de Papadopoulos [13]). Il s'agit maintenant de préciser la loi de comportement couplant plasticité et endommagement, l'objectif étant de relier les variables de déformations à la contrainte définie à l'échelle du grain.

\subsubsection{Couplage plasticité-endommagement à l'échelle du grain}

On considère maintenant la croissance de cavités dans un milieu plastique représentant une BGP. Pour cela, on adopte le cadre de l'analyse limite considéré par Gurson [10]. Compte tenu des difficultés liées à la prise en compte du caractère anisotrope du comportement plastique cristallin, une première étape consiste à substituer au monocristal, régi par la loi de Schmid, un matériau équivalent de von Mises. On considère alors une cellule élémentaire (cf. Fig. 1) définie par une sphère creuse de rayon $b$ contenant une cavité sphérique de rayon $a$ (ce qui définit ainsi une porosité $\left.f=(a / b)^{3}\right)$. Cette sphère est soumise à un taux de déformation uniforme sur son bord extérieur :

$$
\underline{v}(r=b)=D \cdot \underline{x}
$$

$\underline{v}$ étant le champ de vitesse dans la matrice solide. $\boldsymbol{D}$ se compose d'un glissement simple dont l'orientation est donné par $\boldsymbol{\Delta}$ (cf. Éq. (1)), et d'un taux de déformation de type hydrostatique, i.e. $\boldsymbol{D}=\dot{\gamma} \boldsymbol{\Delta}+\dot{\varepsilon}_{\mathrm{h}}^{\mathrm{p}} \mathbf{1}$.

Reconduisant l'approche de Gurson sur la cellule élémentaire, pour les conditions aux limites particulières adoptées, une expression explicite du critère de plasticité a été obtenue (cf. [14] pour plus de détails) et s'écrit :

$$
\mathcal{F}=\frac{(\boldsymbol{\sigma}: \boldsymbol{\Delta})^{2}}{\tau_{0}^{2}}+2 f \cosh \left\{\frac{\sqrt{3}}{2} \frac{\sigma_{\mathrm{h}}}{\tau_{0}}\right\}-1-f^{2}
$$

Comme pour le critère classique de Gurson, (9) fait clairement apparaître que la porosité $f$ affecte la plasticité macroscopique du milieu. Il montre également une dépendance avec la pression hydrostatique. De même,

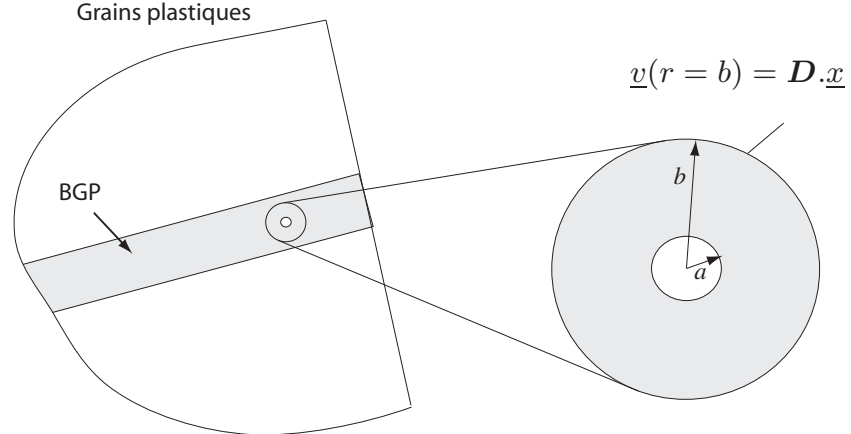

Fig. 1. Cellule élémentaire considérée.

lorsque la porosité est nulle $(f=0)$, on retrouve naturellement le critère de Schmid, en plasticité parfaite, pour le système de glissement prédominant considéré.

On se propose maintenant d'étendre (9), établi dans le cadre de la plasticité parfaite, à la prise en compte de l'écrouissage isotrope et cinématique.

Une extension du critère original de Gurson a été proposée par Leblond et al. [11] en s'appuyant sur le cadre micromécanique ayant permis d'établir le critère de Gurson. Conformément à [11], on suppose que l'expression du critère de plasticité macroscopique avec prise en compte de l'écrouissage isotrope et cinématique s'écrit sous la forme suivante :

$$
\mathcal{F}=\left(\frac{\boldsymbol{B}: \boldsymbol{\Delta}}{\tau_{\mathrm{d}}}\right)^{2}+2 f \cosh \left\{\frac{\sqrt{3}}{2} \frac{B_{\mathrm{h}}}{\tau_{\mathrm{h}}}\right\}-1-f^{2}
$$

où $\boldsymbol{B}=\boldsymbol{\sigma}-\boldsymbol{X}, \boldsymbol{X}$ étant la variable d'écrouissage cinématique définissant le centre du domaine élastique. $\boldsymbol{X}$ se décompose en une partie hydrostatique $X_{\mathrm{h}}\left(X_{\mathrm{h}}=\right.$ $\operatorname{tr}(\boldsymbol{X}) / 3$ ) et une composante de glissement sur le système prédominant, notée $X_{\mathrm{d}}$ et définie par : $X_{\mathrm{d}}=2 \boldsymbol{X}: \boldsymbol{\Delta}$. La prise en compte de l'écrouissage isotrope se fait en remplaçant le seuil de plasticité $\tau_{0}$ par deux paramètres $\tau_{\mathrm{d}}$ et $\tau_{\mathrm{h}}$, définis par : $\tau_{\mathrm{d}}=\tau_{0}+R_{\mathrm{d}}$ et $\tau_{\mathrm{h}}=\tau_{0}+R_{\mathrm{h}}$. Les quantités $R_{\mathrm{d}}$ et $R_{\mathrm{h}}$ sont les deux variables d'écrouissage isotrope. Le problème se réduit donc à la détermination des paramètres $X_{\mathrm{d}}, X_{\mathrm{h}}, R_{\mathrm{d}}$ et $R_{\mathrm{h}}$.

Les expressions des paramètres d'écrouissage sont déterminées en considérant des trajets de chargement pour lesquels une solution exacte du problème d'analyse limite, dans le cas d'une matrice écrouissable, peut être identifiée. Cette méthode, adaptée dans [14] au cas de la plasticité cristalline, conduit aux expressions suivantes pour les paramètres d'écrouissage introduits dans (10) :

$$
\begin{aligned}
& R_{\mathrm{d}}=R_{0} \gamma_{\text {cum }} ; \quad X_{\mathrm{d}}=(1-f) c \gamma ; \quad X_{\mathrm{h}}=\frac{2 c}{\sqrt{3}} \alpha ; \\
& R_{\mathrm{h}}=-\frac{R_{0}}{\ln (f)} \alpha_{\mathrm{cum}}
\end{aligned}
$$

$X_{\mathrm{h}}$ et $R_{\mathrm{h}}$ font intervenir la variable $\alpha$ définie par :

$$
\alpha=\frac{2}{\sqrt{3}}\left[\operatorname{dilog}\left(\frac{f_{\mathrm{a}}}{f}\right)-f_{\mathrm{g}}\right]
$$

et $\alpha_{\text {cum }}=\int_{0}^{t}|\dot{\alpha}| \mathrm{d} t^{\prime}$ est la valeur cumulée de la variable $\alpha$. 

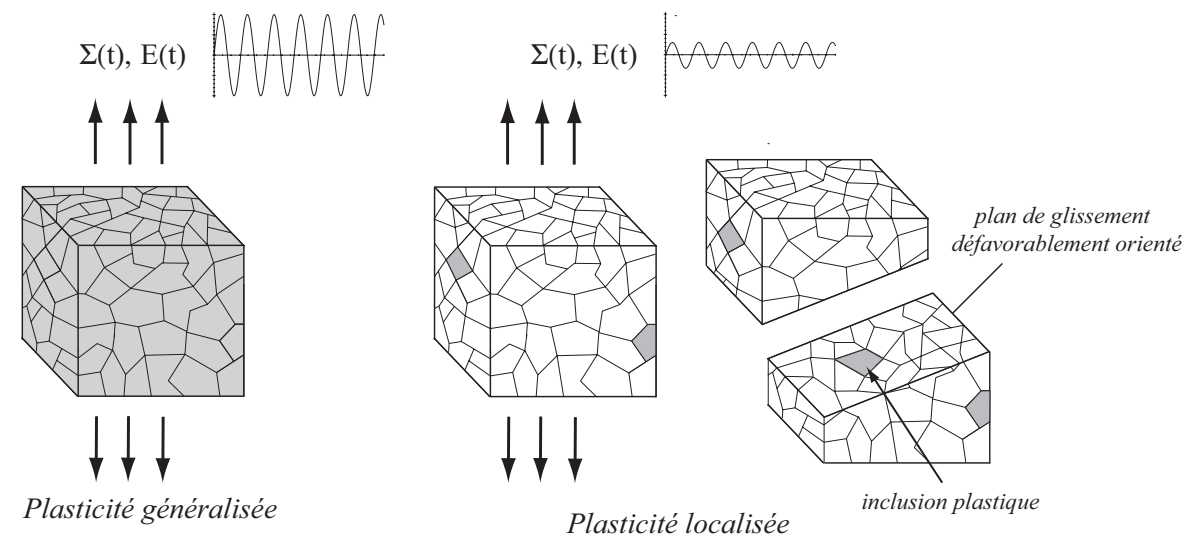

Fig. 2. Représentation schématique de l'état de déformation plastique à l'échelle du VER en fatigue oligocyclique et polycyclique.

\subsubsection{Lois d'évolution}

Les lois d'évolution des variables $\gamma_{\text {cum }}$ et $\varepsilon_{\mathrm{h}}^{\mathrm{p}}$, qui interviennent explicitement dans l'expression du critère de fatigue (7), sont obtenues via la loi de normalité associée à $\mathcal{F}(10)$ :

$$
\dot{\boldsymbol{\varepsilon}}^{\mathrm{p}}=\dot{\lambda} \frac{\partial \mathcal{F}}{\partial \boldsymbol{\sigma}} \Rightarrow\left\{\begin{array}{l}
\dot{\gamma}_{\text {cum }}=2 \dot{\lambda} \frac{|\boldsymbol{B}: \boldsymbol{\Delta}|}{\tau_{\mathrm{d}}^{2}} \\
\dot{\varepsilon}_{\mathrm{h}}^{\mathrm{p}}=\dot{\lambda} \frac{f}{\sqrt{3} \tau_{\mathrm{h}}} \sinh \left\{\frac{\sqrt{3}}{2} \frac{B_{\mathrm{h}}}{\tau_{\mathrm{h}}}\right\}
\end{array}\right.
$$

$\dot{\lambda}$ désignant le multiplicateur plastique. Ces lois d'évolution (13) de $\gamma_{\text {cum }}$ et $\varepsilon_{\mathrm{h}}^{\mathrm{p}}$ font intervenir la contrainte locale et plus précisément $\tau$ et $\sigma_{\mathrm{h}}$. Il s'agit donc maintenant de formuler le critère de fatigue à l'échelle macroscopique, c'est-à-dire en fonction de la contrainte macroscopique, $\boldsymbol{\Sigma}$; ceci requiert un passage micro-macro.

\section{Passage micro-macro}

Dans le cadre de la fatigue polycyclique, on rappelle que la plasticité reste confinée dans quelques grains défavorablement orientés par rapport aux axes de chargement et que le comportement macroscopique, qui n'est pas affecté par cette plasticité, demeure élastique (cf. Fig. 2). Notons par $\boldsymbol{\Sigma}$ le tenseur des contraintes macroscopiques et par $\boldsymbol{E}$ le tenseur des déformations macroscopiques. Ces grandeurs sont reliées par la loi de Hooke : $\boldsymbol{\Sigma}=\mathbb{C}: \boldsymbol{E}$, où $\mathbb{C}$ est le tenseur de rigidité de l'agrégat, que nous supposerons isotrope par simplicité. Le comportement élastique de l'inclusion (le grain sollicité) est défini par $\sigma=\mathbb{C}^{I}: \varepsilon^{\mathrm{e}}$. En toute généralité, on a $\mathbb{C} \neq \mathbb{C}^{I}$, puisque le comportement élastique du grain est généralement caractérisé par une anisotropie de type cubique; néanmoins, par souci de simplicité, on néglige cette anisotropie et on pose $\mathbb{C}=\mathbb{C}^{I 1}$.

\footnotetext{
1 Notons toutefois que la prise en compte de l'anisotropie cubique du grain ne pose aucune difficulté supplémentaire, elle a d'ailleurs été traitée dans [14].
}

Le cas de figure de la fatigue polycyclique peut se rapprocher de celui de l'entrée en plasticité (plasticité confinée) des métaux pour laquelle l'utilisation d'une approche de type Eshelby-Kröner est pleinement justifiée (cf. [15]). En reprenant le schéma autocohérent de Kröner [12], on a la loi d'interaction :

$$
\sigma=\Sigma-\mathbb{C}^{*}: \varepsilon^{\mathrm{p}}
$$

où $\mathbb{C}^{*}=\mathbb{C}:(\mathbb{I}-\mathbb{P}: \mathbb{C}), \mathbb{P}$ est le tenseur d'ordre quatre de Hill, qui dépend des propriétés élastiques du milieu environnant, et de la géométrie de l'inclusion. Pour des inclusions sphériques, plongées dans un milieu élastique isotrope infini, celui-ci s'écrit classiquement :

$$
\begin{aligned}
\mathbb{P} & =\frac{\kappa_{1}}{3 k} \mathbb{J}+\frac{\kappa_{2}}{2 \mu} \mathbb{K} \quad \text { avec }: \kappa_{1}=\frac{3 k}{3 k+4 \mu}=\frac{1}{3} \frac{1+\nu}{1-\nu} \quad \text { et } \\
\kappa_{2} & =\frac{6}{5} \frac{k+2 \mu}{3 k+4 \mu}=\frac{2}{15} \frac{4-5 \nu}{1-\nu}
\end{aligned}
$$

où $\nu$ est le coefficient de Poisson de l'agrégat. Il s'ensuit pour la pression hydrostatique, $\sigma_{\mathrm{h}}$, et la cission, $\tau$, qui interviennent dans (9), (13) et donc pour la détermination du critère de fatigue (7) :

$$
\tau=\boldsymbol{\Sigma}: \Delta-\mu^{*} \gamma ; \quad \sigma_{\mathrm{h}}=\Sigma_{\mathrm{h}}-3 k^{*} \varepsilon_{\mathrm{h}}^{\mathrm{p}}
$$

avec :

$$
k^{*}=\frac{2 k}{3} \frac{1-2 \nu}{1-\nu} \quad \text { et } \quad \mu^{*}=\frac{\mu}{15} \frac{7-5 \nu}{1-\nu}
$$

La transposition de la loi de comportement locale, définie par (10) et (13), à l'échelle macroscopique est obtenue à partir de (16) et conduit tout simplement à définir un écrouissage apparent, noté $X_{\mathrm{d}}^{*}=X_{\mathrm{d}}+\mu^{*} \gamma$ et $X_{\mathrm{h}}^{*}=X_{\mathrm{h}}+$ $3 k^{*} \varepsilon_{\mathrm{h}}^{\mathrm{p}}$. On obtient ainsi pour la surface de charge (10) :

$$
\mathcal{F}=\left(\frac{\boldsymbol{\Sigma}: \Delta-X_{\mathrm{d}}^{*}}{\tau_{\mathrm{h}}}\right)^{2}+2 f \cosh \left\{\frac{\sqrt{3}}{2} \frac{\Sigma_{\mathrm{h}}-X_{\mathrm{h}}^{*}}{\tau_{\mathrm{h}}}\right\}-1-f^{2}
$$


et pour les lois d'évolutions des variables de déformations plastiques :

$$
\begin{aligned}
\dot{\gamma}_{\text {cum }} & =2 \dot{\lambda} \frac{\left|\boldsymbol{\Sigma}: \Delta-X_{\mathrm{d}}^{*}\right|}{\tau_{\mathrm{d}}^{2}} \\
\dot{\varepsilon}_{\mathrm{h}}^{\mathrm{p}} & =\dot{\lambda} \frac{f}{\sqrt{3} \tau_{\mathrm{h}}} \sinh \left\{\frac{\sqrt{3}}{2} \frac{\Sigma_{\mathrm{h}}-X_{\mathrm{h}}^{*}}{\tau_{\mathrm{h}}}\right\}
\end{aligned}
$$

On notera que du point de vue de l'intégration numérique du modèle, la transposition à l'échelle macroscopique n'introduit aucune difficulté supplémentaire. De manière générale la contrainte macroscopique est une fonction du temps, décrivant dans l'espace des contraintes une courbe fermée correspondant au trajet de chargement cyclique. La détermination du critère de fatigue peut alors être effectuée en intégrant la loi de comportement définie par (18) sur le trajet de chargement. Il s'agit alors de vérifier la condition de non amorçage d'une fissure de fatigue (7).

À titre d'illustration on propose une application du critère obtenu dans le cas des trajets de chargement affines macroscopiques.

\section{Application aux trajets de chargement affines}

\subsection{Mise en œuvre du modèle pour les trajets affines}

Lorsque le trajet de chargement macroscopique est affine, le tenseur des contraintes macroscopiques s'écrit :

$$
\boldsymbol{\Sigma}(t)=\boldsymbol{\Sigma}_{\mathrm{a}} \sin (\omega t)+\boldsymbol{\Sigma}_{\mathrm{m}}
$$

où $\boldsymbol{\Sigma}_{\mathrm{a}}$ et $\boldsymbol{\Sigma}_{\mathrm{m}}$ désignent respectivement les parties alternées et moyennes du tenseur des contraintes macroscopiques dont les composantes de scission sont notées $T_{\mathrm{a}}=\boldsymbol{\Sigma}_{\mathrm{a}}: \boldsymbol{\Delta}$ et $T_{\mathrm{m}}=\boldsymbol{\Sigma}_{\mathrm{m}}: \boldsymbol{\Delta}$, et les composantes hydrostatiques, $\Sigma_{\mathrm{h}, \mathrm{a}}$ et $\Sigma_{\mathrm{h}, \mathrm{m}}$.

Dans le cas des trajets de chargement macroscopiques affines, le comportement asymptotique des matériaux plastiques se caractérise par l'adaptation, l'accommodation ou le rochet. Dans les deux derniers cas, la rupture survient par accumulation des déformations plastiques et donc du dommage le long des BGP. Une condition nécessaire de non rupture consiste alors à assurer l'adaptation à l'échelle locale. Dans le cas d'un écrouissage isotrope la réponse asymptotique du matériau se caractérise par l'adaptation. On propose dans cette première soussection de présenter des résultats numériques obtenus à partir du modèle d'endommagement décrit dans les sections précédentes, dans le cas des chargements affines. Pour les différentes applications proposées, les paramètres du modèle utilisés sont donnés dans le tableau 1 .

Le critère de plasticité à l'état adapté (saturé) dans l'espace des contraintes macroscopiques $\left(\boldsymbol{\Sigma}: \boldsymbol{\Delta}, \Sigma_{\mathrm{h}}\right)$ est représenté sur la figure 3 pour les valeurs particulières suivantes : $T_{\mathrm{a}}=200 \mathrm{MPa}, T_{\mathrm{m}}=0, \Sigma_{\mathrm{h}, \mathrm{a}}=600 \mathrm{MPa}$ et $\Sigma_{\mathrm{h}, \mathrm{m}}=200 \mathrm{MPa}$. On note que la surface de charge est symétrisée sur le cycle des contraintes macroscopiques

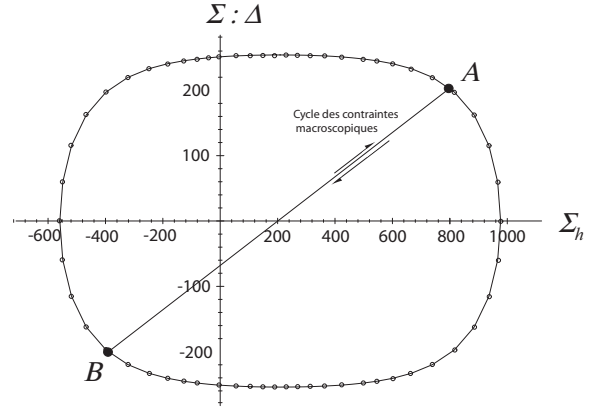

Fig. 3. Surface de charge dans le plan $\left(\boldsymbol{\Sigma}: \boldsymbol{\Delta}, \Sigma_{\mathrm{h}}\right)$.

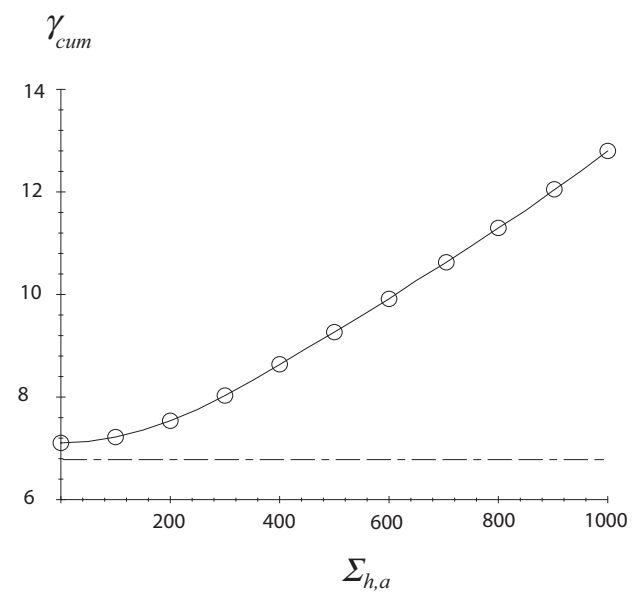

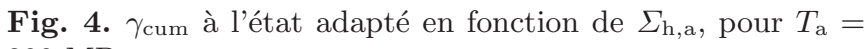
$200 \mathrm{MPa}$.

(schématisé par le segment $A B$ ), c'est-à-dire autour de $T_{\mathrm{m}}=0$ et $\Sigma_{\mathrm{h}, \mathrm{m}}=200 \mathrm{MPa}$. Cet aspect a pu être vérifié de manière générale : en fait à l'échelle locale du grain, à l'état adapté, le cycle des contraintes est défini par $\boldsymbol{\sigma}=$ $\boldsymbol{\Sigma}_{\mathrm{a}} \sin (\omega t)$; les contraintes résiduelles s'équilibrent alors avec le tenseur des contraintes moyennes macroscopiques. On a donc $\boldsymbol{\rho}=\boldsymbol{\Sigma}_{\mathrm{m}}$. Les trois relations suivantes sont alors établies :

$$
\begin{gathered}
T_{\mathrm{m}}=X_{\mathrm{d}}^{*}=\left(\left(1-f_{\mathrm{c}}\right) c+\mu^{*}\right) \gamma \\
\Sigma_{\mathrm{h}, \mathrm{m}}=X_{\mathrm{h}}^{*} \\
\frac{T_{\mathrm{a}}^{2}}{\tau_{\mathrm{d}}^{2}}+2 f_{\mathrm{c}} \cosh \left\{\frac{\sqrt{3}}{2} \frac{\Sigma_{\mathrm{h}, \mathrm{a}}}{\tau_{\mathrm{h}}}\right\}-1-f_{\mathrm{c}}^{2}=0
\end{gathered}
$$

Notons que le résultat qui vient d'être souligné étend celui déjà obtenu par Papadopoulos [13] en plasticité classique au cas d'une plasticité dépendant de la pression hydrostatique. Sur la figure 4 sont tracées les valeurs du glissement plastique $\gamma_{\text {cum }}$ en fonction de l'amplitude de la pression hydrostatique $\Sigma_{\mathrm{h}, \mathrm{a}}$, pour une amplitude du cisaillement $T_{\mathrm{a}}=200 \mathrm{MPa}$. La variation de $\gamma_{\text {cum }}$ avec cette composante de la pression hydrostatique illustre bien la dépendance du comportement plastique vis-à-vis de $\Sigma_{\mathrm{h}}$ et donc l'importance du couplage entre la plasticité et l'endommagement.

Sur la figure 5, est tracée la variation de la porosité, $f$, en fonction du nombre de cycles. On observe encore une dépendance nette de la valeur de la porosité 
Tableau 1. Paramètres utilisés pour le modèle.

\begin{tabular}{|c|c|c|c|c|c|c|}
\hline$\tau_{0}$ & $R_{0}$ & $c$ & $A_{0}$ & $k_{\mathrm{a}}$ & $k$ & $\mu$ \\
\hline $60 \mathrm{MPa}$ & $20 \mathrm{MPa}$ & $1000 \mathrm{MPa}$ & 0,0008 & 2 & $200 \mathrm{GPa}$ & $75 \mathrm{GPa}$ \\
\hline
\end{tabular}

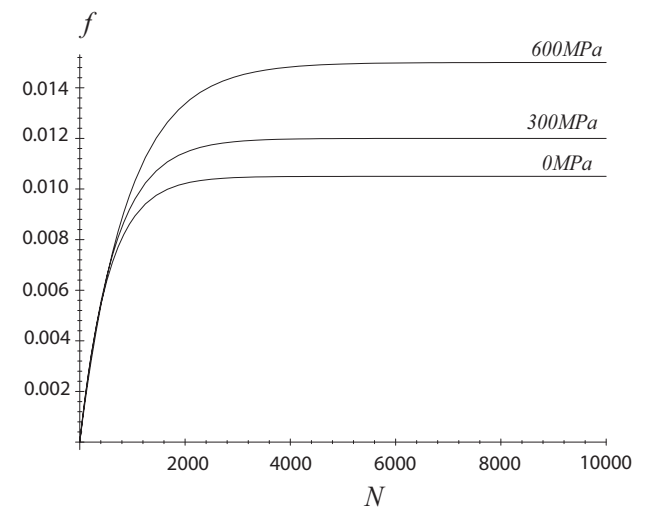

Fig. 5. Valeur de $f$ en fonction du nombre de cycles $N$, pour le schéma de Kröner et pour diverses valeurs de $\Sigma_{\mathrm{h}, \mathrm{a}}=0 \mathrm{MPa}$, $300 \mathrm{MPa}, 600 \mathrm{MPa}$.

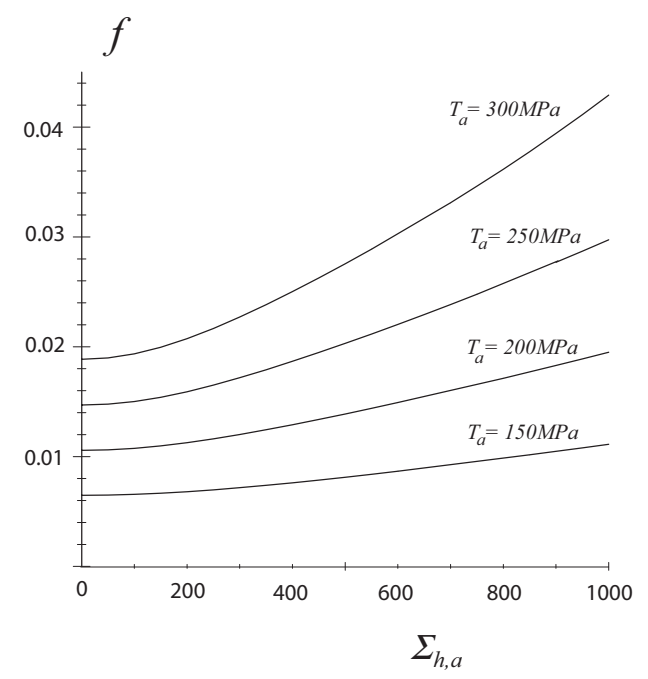

Fig. 6. Variation de la porosité $f$ avec l'amplitude de la pression hydrostatique $\Sigma_{\mathrm{h}, \mathrm{a}}$ pour diverses valeurs de l'amplitude du cisaillement $T_{\mathrm{a}}$.

vis-à-vis de l'amplitude de la pression hydrostatique, $\Sigma_{\mathrm{h}, \mathrm{a}}$ qui, encore une fois, résulte du couplage de la surface de charge avec l'endommagement et la pression hydrostatique. Cette dépendance est également observée sur la figure 6 , sur laquelle est tracée la variation de la porosité, $f$, avec $\Sigma_{\mathrm{h}, \mathrm{a}}$ pour diverses valeurs de l'amplitude du cisaillement $T_{\mathrm{a}}$. Sur la figure 7 , est tracée la variation de la porosité, $f$, en fonction de la valeur de $\Sigma_{\mathrm{h}, \mathrm{m}}$ pour diverses valeurs de $T_{\mathrm{a}}$. On observe également une dépendance de la porosité vis-à-vis de la pression hydrostatique moyenne.

\subsection{Détermination du critère de fatigue macroscopique}

Nous avons pu vérifier par résolution numérique de la loi de comportement que pour le type de trajet de

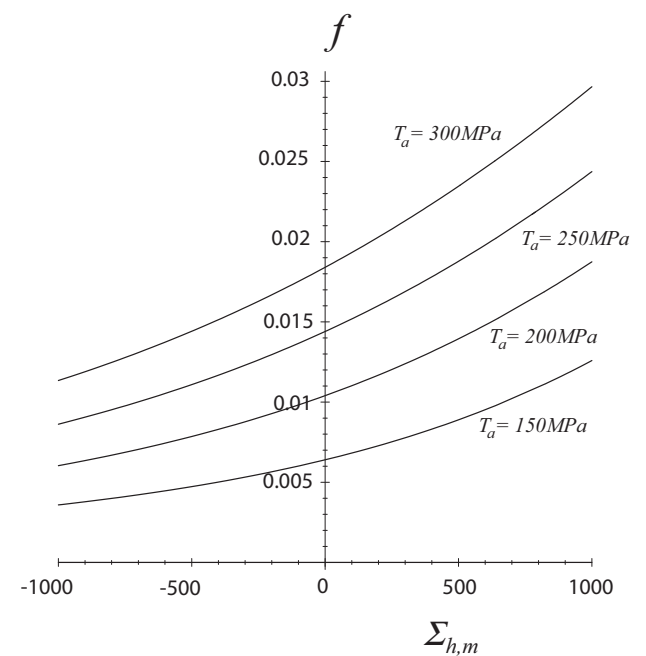

Fig. 7. Variation de la porosité $f$ avec la moyenne de la pression hydrostatique $\Sigma_{\mathrm{h}, \mathrm{m}}$ pour diverses valeurs de l'amplitude du cisaillement $T_{\mathrm{a}}$.

chargement considéré ici, à l'adaptation, le cycle de contrainte microscopique est symétrisé. Ceci nous a permis d'établir les trois relations données par (21). On notera tout d'abord que le critère de fatigue est fonction seule de $\gamma_{\text {cum }}$ et $\varepsilon_{\mathrm{h}}^{\mathrm{p}}$ mais ne fait pas intervenir la valeur du glissement plastique à l'état adapté, $\gamma$. Ceci implique, compte tenu de la première relation dans (21), que le critère est indépendant de la cission moyenne macroscopique; ceci est en accord avec des résultats expérimentaux de la littérature [3]. Des deux dernières relations dans (21) on peut déduire les variables locales $\gamma_{\text {cum }}$ et $\varepsilon_{\mathrm{h}}^{\mathrm{p}}$ en fonction des grandeurs macroscopiques $T_{\mathrm{a}}, \Sigma_{\mathrm{h}, \mathrm{a}}, \Sigma_{\mathrm{h}, \mathrm{m}}$. On notera de manière générale que le critère proposé fait donc intervenir les même variables que le critère de Dang Van. Toutefois, la dépendance avec les composantes alternées et moyennes de la pression hydrostatique, $\Sigma_{\mathrm{h}, \mathrm{a}}$ et $\Sigma_{\mathrm{h}, \mathrm{m}}$, résulte clairement ici de la prise en compte des mécanismes d'endommagement survenant le long des BGP.

À titre d'illustration on propose deux comparaisons du critère de fatigue proposé avec des données expérimentales issues de la littérature. Sur la figure 8 est considérée une comparaison du critère dans le cas d'une torsion alternée, $\Sigma_{12 \text {,a }}$, superposée avec une traction moyenne, $\Sigma_{11, \mathrm{~m}}$. On observe que le critère, représenté dans le plan $\left(\Sigma_{12, \mathrm{a}} ; \Sigma_{11, \mathrm{~m}}\right)$, permet de bien reproduire l'effet d'une pression moyenne (représentée par $\Sigma_{11, \mathrm{~m}}$ ). Sur la figure 9 est proposée une comparaison avec les essais de Gough et Pollard [6] en flexion-torsion alternée. Le critère, représenté dans le plan $\left(\Sigma_{12, \mathrm{a}} ; \Sigma_{11, \mathrm{a}}\right)$, permet à nouveau de bien reproduire l'effet d'une pression hydrostatique alternée. 
Amplitude de la torsion : $\Sigma_{12, a}($ en $\mathrm{MPa})$

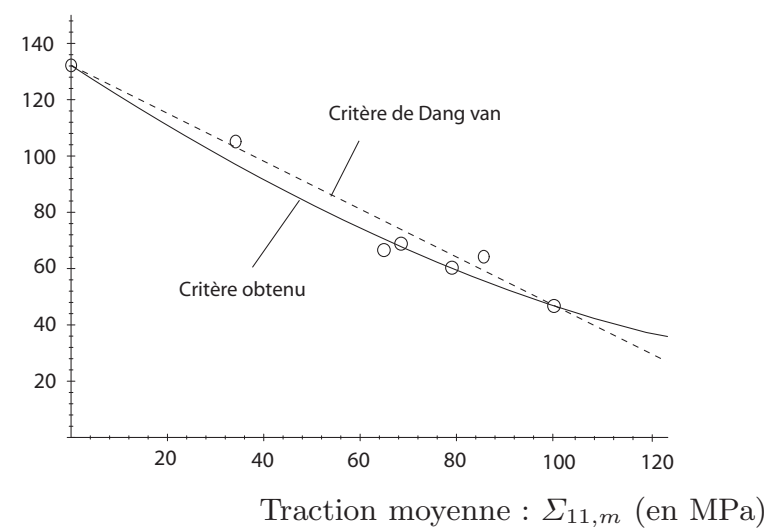

Fig. 8. Torsion alternée-traction moyenne : comparaison du critère de fatigue obtenu avec celui de Dang Van et les données expérimentales de Rõs, issues de [8] (cercles).

Amplitude de la torsion : $\Sigma_{12, \mathrm{a}}(\mathrm{en} \mathrm{MPa})$

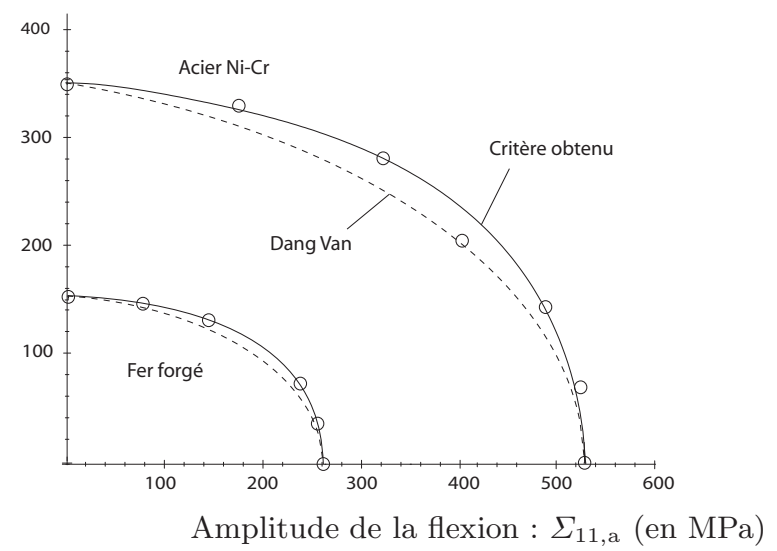

Fig. 9. Flexion-torsion alternée : comparaison du critère de fatigue obtenu avec celui de Dang Van et les données expérimentales de Gough et Pollard [16] (cercles).

\section{Conclusion}

Une approche multiéchelle pour la détermination d'un critère de fatigue à grand nombre de cycles a été proposée. Elle permet de prendre en compte, non seulement l'activité plastique de certains grains, mais également l'endommagement dû à la croissance de microcavités à l'interface BGP-matrice. Le critère proposé est établi en adaptant l'extension des travaux de Gurson proposée par Leblond et al., étendue ici aux chargements cycliques et à la plasticité cristalline écrouissable. Cette approche permet de rendre compte de l'important effet de la pression hydrostatique moyenne ainsi que de sa partie alternée, en raison du caractère multiéchelle de la démarche. Cet effet est clairement relié aux mécanismes d'endommagement considérés. Il est certain que l'approche proposée ainsi que les résultats obtenus ouvrent de nouvelles perspectives en fatigue, en particulier au traitement de la plasticité macroscopique et aux chargements cycliques thermomécaniques qui demeurent des problèmes ouverts.

\section{Références}

[1] U. Essmann, H. Mughrabi, Annihilation of dislocations during tensile and cyclic deformation and limits of dislocation densities. Phil. Mag. A 171 (1979) 731-756

[2] K. Dang Van, Introduction to fatigue analysis in mechanical design by the multiscale approach. High-Cycle fatigue in the context of mechanical design, CISM courses and lectures, 392, ed. K. Dang-Van and I.V. Papadopoulos, Springer-Verlag, 1999, pp. 1691-1710

[3] G. Sines, J.L. Waisman, Metal fatigue. McGraw Hill, 1959

[4] G. Sines, G. Ohgi, Fatigue criteria under combined stresses or strains, J. Engrg. Mat. Tech. 103 (1981) 82-90

[5] B. Crossland, Effects of large hydrostatic pressures on tortional fatigue strength of an alloy steel, Proc. Int. Conf. Fatigue of Metals, Institution of Mechanical Engineers, London, 1956, pp. 138-149

[6] E. Orowan, Theory of the fatigue of metals, Proc. Roy. Society, A, London, 40, 1939, pp. 78-106

[7] K. Dang Van, Sur la résistance à la fatigue des métaux, Thèse de doctorat, Université Paris VI, 1973

[8] I.V. Papadopoulos, Fatigue polycyclique des métaux : une nouvelle approche, Thèse de doctorat de l'ENPC, 1987

[9] J.R. Rice, D.M. Tracey, On a ductile enlargement of voids in triaxial stress fields, J. Mech. Phys. Solids 17 (1969) $201-217$

[10] A.L. Gurson, Continuum theory of ductile rupture by void nucleation and growth: part I- yield criteria and flow rules for porous ductile media, J. Engrg. Mater. Technol. 99 (1977) 2-15

[11] J.B. Leblond, G. Perrin, J. Devaux, An improved Gursontype model for hardenable ductile metals, Eur. J. Mech. A/Solids 14 (1995) 499-527

[12] E. Kröner, Zur plastichen verformung des vielkristalls, Acta Metall. 99 (1961) 155-161

[13] I.V. Papadopoulos, Exploring the high-cycle fatigue behaviour of metals from the mesoscopic scale, J. Mech. Behav. Mat. 6 (1996) 93-118

[14] V. Monchiet, Contributions à la modélisation micromécanique de l'endommagement et de la fatigue des métaux ductiles, Thèse de doctorat de l'université de Lille I, 2006

[15] M. Bornert, T. Bretheau, P. Gilormini, Homogénéisation en mécanique des matériaux, Tome 2, Comportements non linéaires et problèmes ouverts, Hermes Science (2001)

[16] H.J. Gough, H.V. Pollard, The strength of metals under combined alternating stress, Proc. Inst. Mech. Engrg. 131 (1935) 3-18

[17] V. Monchiet, E. Charkaluk, D. Kondo, A plasticitydamage based micromechanical modelling in high cycle fatigue, C.R. Mécanique 334 (2006) 129-136

[18] P. Davoli, A. Bernasconi, M. Filippini, S. Foletti, I.V. Papadopoulos, Independence of the torsional fatigue limit upon a mean shear stress, Int. J. Fatigue 25 (2003) 471480 